\title{
LA TRANSIZIONE FALLITA. DIECI ANNI DI POLITICA MOZAMBICANA (2009-2019)
}

di Luca Bussotti

\section{Introduzione}

Per diverso tempo, dopo la firma degli Accordi Generali di Pace (AGP) a Roma, il 4 ottobre del 1992, fra il governo mozambicano guidato dal FRELIMO (Fronte di Liberazione del Mozambico) e la RENAMO (Resistenza Nazionale del Mozambico), una buona parte della comunità internazionale e accademica aveva considerato il Mozambico come un raro caso di successo in Africa.

La realtà si è rivelata assai più complessa rispetto alle previsioni iniziali, nonostante l'illusione di un paese pacificato e sulla via di una democrazia stabile sia stata propagandata a lungo, anche a causa di un incremento medio annuo del PIL pari al 7,2\% fra il 2000 e il $2016^{1}$.

Questo studio offre una riflessione su come la "transizione" dalla Prima alla Seconda Repubblica - ossia da uno Stato socialista con un regime a partito unico a uno democratico e multipartitico, non abbia seguito il percorso che era stato auspicato e previsto durante tutti gli anni Novanta e per buona parte del nuovo secolo. Al contrario, vi è stata una deriva autoritaria associata a nuovi conflitti interni. Non si è trattato di un fenomeno improvviso, ma costante, a partire dal 2009 circa fino a oggi, come tutti i principali indicatori su democrazia, libertà di espressione, diritti umani evidenziano.

Centro de Estudos Avançados e Programa de Pós-Graduação em Sociologia, Universidade Federal de Pernambuco.

1 The World Bank, Moçambique. Avaliação da pobreza, Maputo, The World Bank, 2016, in http://documents1.worldbank.org/curated/en/600731554132409626/pdf/Overview.pdf. 
La tesi che qui si intende dimostrare è che gli elementi di questo fallimento devono essere ricercati nel legame inscindibile fra pace e democrazia, e nell'instaurazione di una pace "negativa" in Mozambico fra le due parti che si erano confrontate per 16 anni in una sanguinosa guerra civile. Nonostante gli accordi di pace e il cambiamento politico-istituzionale, le relazioni fra i due maggiori partiti del paese sono rimaste improntate a quella violenza e reciproca sfiducia che avevano caratterizzato gli anni del conflitto e delle "purghe" dello Stato contro i "nemici", avendo ciascuna delle due parti, come unico obiettivo, l'annullamento dell'altra. Circostanza che, alla fine, si è verificata, con modalità piuttosto complesse, come si dirà più avanti.

Il periodo scelto per questa ricerca va dal 2009 (secondo mandato come presidente della repubblica di Armando Emílio Guebuza) al 2019 (seconde elezioni presidenziali vinte da Filipe Nyusi), un decennio contrassegnato da un regresso della democrazia mozambicana, a causa del modello di governance autoritario imposto da questi due leaders politici, nonostante le notevoli differenze fra di loro.

\section{Breve quadro teorico-metodologico}

Una grande parte dei processi di pacificazione in Africa non ha mai ottenuto gli effetti desiderati. In qualche caso il conflitto non si è arrestato, in altri la sua conclusione è stata così insoddisfacente da creare $\mathrm{i}$ presupposti per ulteriori, nuove tensioni fra diversi gruppi sociali, politici ed etnici. Il risultato, nel migliore dei casi, si è concretizzato in una pace "negativa", spesso associata a regimi anti-democratici.

Il concetto di "pace negativa" - anche in opposizione rispetto alla pace "positiva" - è stato introdotto da Galtung. La locuzione indica il superamento del tipo di violenza su cui si fonda la pace. La pace negativa riesce a eliminare o limitare molto attacchi personali, guerra, terrorismo, disordini di piazza, senza però intaccare alcuni elementi strutturali tipici di una violenza "indiretta", ossia strutturale, come povertà, fame, discriminazione, apartheid ${ }^{2}$. In questo caso, gruppi di solito

2 O. Herath, A Critical Analysis of Positive and Negative Peace, Oshadhi, Department of Philosophy, University of Kelaniya, 2016, in http://repository.kln.ac.lk/bitstream/handle/ $123456789 / 12056 /$ journal $1 \% 20 \% 281 \% 29.104-107$.pdf? sequence=1\&isAllowed=y. 
numericamente minoritari hanno un accesso privilegiato o esclusivo a risorse e benefici, condizionando le politiche pubbliche. Soltanto quando questo genere di violenza che permea i rapporti sociali o etnici di una intera nazione viene superato, è possibile parlare di pace positiva ${ }^{3}$.

Oggi, tale concetto è stato tradotto in un indice globale basato su otto principi fondamentali, fra cui giustizia, tolleranza e assenza di corruzione $^{4}$. Nelle top-30 posizioni del Global Peace Index non vi è alcun paese africano, mentre ve ne sono 6 fra gli ultimi $10^{5}$.

La ricerca di un risultato immediato e tangibile (l'accordo di pace firmato) di solito caratterizza il modus operandi dei mediatori internazionali, trascurando il fatto che la fiducia reciproca è l'aspetto centrale che sta alla base dell'instaurazione di una pace positiva ${ }^{6}$. Meccanismi di riconciliazione nazionale, come commissioni per ristabilire la verità storica secondo quanto accaduto, per esempio, in Sud Africa, costituiscono elementi essenziali per il superamento della logica di vendetta e di violenza che, altrimenti, permane immutata anche se in uno scenario politico diverso ${ }^{7}$. Senza l'attivazione di tali meccanismi, la probabilità di instaurare una pace negativa è assai elevata, dando luogo a una democrazia debole, un elevato livello di autoritarismo, intolleranza e corruzione, col concreto rischio di un nuovo conflitto ${ }^{8}$. Lo stesso caso del Sud Africa è sintomatico in questo senso: benché dopo la fine dell'apartheid si riuscì ad evitare un'escalation di violenza, questo è ancora il paese col più alto coefficiente di Gini al mondo. Ciò è dovuto al fatto che si sono mantenuti gli elementi di violenza strutturale (diseguaglianza al massimo livello) del periodo dell'apartheid, fatto che ha portato, ciclicamente, a rivolte, manifestazioni violente (l'ultima delle quali in reazione all'arresto dell'ex-presidente Zuma, a luglio del

3 J. Galtung, An Introduction, in "Journal of Peace Research", n. 1, 1964, pp. 1-4.

4 Positive Peace Academy, Discover the 8 Pillars of Positive Peace, 2020, in https://positivepeace.academy/.

5 Institute for Economics \& Peace, Global Peace Index 2020, in http://visionofhumanity.org/app/uploads/2020/06/GPI_2020_web.pdf.

6 V. Adetula, T. Murithi, \& S. Buchanan-Clarke, Peace negotiations and agreements in Africa: why they fail and how to improve them, Uppsala, The Nordic African Institute, in http://nai.diva-portal.org/smash/record.jsf?pid=diva2\%3A1262987\&dswid=1408.

7 Sul caso sudafricano, fra la vasta letteratura vedasi J. BranKovic, Responsabilidad y Reconciliación Nacional en Sudáfrica, in "Ediciones Infojus: Derechos Humanos", n. 4, 2013, pp. 55-86.

8 J. Galtung, Theories of Peace, Oslo, International Peace Research Institute, 1967 
2021), xenofobia contro gli immigrati di origine africana. Non si può, quindi, affermare che in questo paese vi sia una pace positiva.

Il Mozambico rappresenta un altro caso emblematico di un paese raffigurato come esempio di successo in termini di pacificazione, ma che mai ha risolto le sue antiche questioni di violenza strutturale, soprattutto nel centro e nel nord del proprio territorio, come hanno fra gli altri documentato Muchemwa e Harris 9 . Qui, maggioranze etniche (nel centro Nyanja, Sena e Ndau, nel nord Makhuwa e Kimwani) sono state sopraffatte da minoranze, in particolare dallo storico vincolo fra Makonde (una etnia minoritaria nella sua stessa provincia, Cabo Delgado, ma che ha avuto il merito storico di costituire la più numerosa truppa dell'esercito di liberazione) e Ronga (etnia del sud, che ha fornito tutti i leaders politici del movimento di liberazione, da Mondlane a Machel a Chissano), che ha portato all'indipendenza del Mozambico dal Portogallo. L'accesso alle ingenti risorse naturali, soprattutto del sottosuolo, nonché ai benefici (come pensioni ad hoc, borse di studio per l'estero, concessioni e licenze varie) riservati agli antichi combattenti, quasi tutti Makonde e alle cariche pubbliche hanno sistematicamente discriminato le suddette etnie del centro e del nord, creando una situazione di strutturale ingiustizia, che è sfociata non soltanto nella guerra dei 16 anni tra governo e RENAMO, poi ripresa intorno al 2013 e ancora oggi non completamente risolta, ma anche nell'inaspettata ribellione di matrice islamica a Cabo Delgado ${ }^{10}$. ̇̀ questo tipo di pace che è stato instaurato in Mozambico con gli accordi del 1992 a costituire il terreno di fondo di conflitti interni costanti, diversi fra loro, ma con una matrice comune: il tentativo di ribellarsi alla sistematica esclusione da privilegi economici e politici da parte di un partito-stato fortemente caratterizzato sul piano etnico.

Nonostante la mediazione della Comunità di Sant'Egidio e dello Stato italiano, che aveva portato agli Accordi Generali di Pace di Roma,

9 C. Muchemwa e G.T.Harris, Mozambique's post-war success story: It is time to revisit this narrative?, in "Democracy and Security", n. 1, 2019, pp. 25-48

${ }^{10} \mathrm{Cfr}$. su questo, fra gli altri: E. Morier-Genoud, The Jihadi Insurgency in Mozambique: Origins, Nature and Beginning, in "Journal of Eastern African Studies", n. 3, 2020, pp. 396-412, e S. Habibe, S. Forquilha e J. PereIra, Radicalização islâmica no Norte de Moçambique, Maputo: IESE, in https://www.iese.ac.mz/wp-content/uploads/2019/09/cadernos_17.pdf 
ritenuti dai più come un modello da applicare anche ad altri casi africani ${ }^{11}$, molti dei provvedimenti previsti non sono stati rispettati: il disarmo e soprattutto la reintegrazione nella vita civile dei militari della RENAMO sono stati assai parziali, anche giocando sul fatto che il suo leader, al tempo Afonso Dhlakama, ha di fatto trasformato il diritto alla scorta personale in un vero e proprio esercito, con tanto di basi militari nel centro del paese; l'apparato dello Stato ha continuato a rispondere alla logica del FRELIMO come partito unico, secondo un modello ad excludendum rispetto a chi non appartenesse a quella formazione politica; il presidente della repubblica ha continuato a godere di poteri amplissimi, senza soluzione di continuità rispetto a quanto avveniva in epoca socialista, con un'oggettiva assenza di una nitida divisione fra i poteri dello stato; infine, i processi elettorali sono stati sempre viziati da enormi frodi da parte del FRELIMO, ignorando sistematicamente la volontà popolare. Infine, le disuguaglianze sono cresciute, sia a livello sociale che territoriale, con un malessere diffuso specialmente nel centro e nel nord del paese. Tutto ciò ha generato sfiducia e malessere generalizzati che si sono tradotti, sul piano elettorale, in un crescente astensionismo, e su quello sociale nelle ricordate rivolte e guerre civili.

I momenti centrali e decisivi per l'inversione della transizione mozambicana da democratica ad autoritaria vanno ricercati, all'interno del quadro di violenza strutturale permanente, in alcuni elementi specifici che hanno fatto precipitare il già labile quadro politico-sociale del paese. Fra questi, processi elettorali opachi e viziati da gravissime frodi, il tipo di governance proposto prima da Armando Emílio Guebuza, subentrato nel 2004 a Joaquim Chissano come presidente della repubblica, e poi da Filipe Nyusi, la concentrazione delle ricchezze economiche nelle mani di un ristretta élite politica. In quest'ultimo caso, per la prima volta in modo così esplicito, la governance mozambicana è stata caratterizzata da una logica etnica, tutta spostata a favore dei Makonde, già egemoni nella provincia di Cabo Delgado. Tale processo è stato favorito dalla congenita debolezza della società civile locale $^{12}$, schiacciata fra controllo del partito-stato FRELIMO e dipendenza

${ }^{11}$ R. Morozzo Della Rocca, Dalla guerra alla pace. Storia di una mediazione insolita, Cinisello Balsamo, Edizioni San Paolo, 1994.

12 Sulla società civile nei paesi in via di transizione democratica, cfr. J. MANOR, Rethinking third world politics, Harlow, Longman, 1991. 
della propria agenda dagli interessi dei donatori internazionali, sviluppando quindi livelli di autonomia modesti e non in grado di contrastare i meccanismi di frode elettorale normalmente usati in Mozambico ${ }^{13}$.

Per questo, come proposto da Ronceray e Byires, in termini metodologici vengono qui analizzati tre elementi centrali del decennio 20092019: i momenti propriamente elettorali, concentrati nelle elezioni presidenziali, legislative e provinciali del 2009, 2014 e 2019; i momenti pre-elettorali, relativi alla preparazione delle elezioni e al relativo clima politico; e i momenti post-elettorali, di gestione dei risultati e loro conseguenze ${ }^{14}$.

Sulla base di questo modello, si è suddiviso il lavoro in tre brevi periodi, prima di giungere alle considerazioni finali: il primo cerca di interpretare il clima politico precedente al 2009 come premessa per l'inversione della transizione, da democratica ad autoritaria; il secondo va dal 2009 al 2014, e viene qui considerato come l'inizio dell'ascesa del modello autoritario; il terzo va dal 2014 al 2019 ed è stato qui definito come il consolidamento dell'autoritarismo mozambicano, mediante la "makondizzazione" dello Stato, a cui è succeduto il clima di tensione e il duplice conflitto in atto oggi nel paese.

2. L'inversione della transizione democratica: la preparazione delle elezioni del 2009

Le elezioni del 2009 si aprivano sotto buoni auspici per il FRELIMO, anche se con alcuni dubbi: in primo luogo, il ricordo dei risultati delle due elezioni precedenti era ancora vivo nella memoria collettiva del partito e del paese. Nel 2004, infatti, dopo diciotto anni di presidenza di Chissano, Guebuza era stato eletto presidente, ma senza raggiungere la maggioranza qualificata, fermandosi al $63 \%$ dei voti. Di contro, la

${ }^{13}$ Sulla società civile mozambicana si veda A. Francisco, Sociedade civil em Moçambique, in IESE, "Desafios para Moçambique 2010", Maputo, 2009, pp. 51-106, https://biblioteca.isced.ac.mz/bitstream/123456789/1038/1/SOCIEDADE\%20CIVIL\%20E M\%20MO\%C3\%87AMBIQUE.pdf.

${ }^{14}$ M. RONCERAY, \& B. BYIERS, Elections in Africa - Playing the game or bending the rules?, "Discussion Paper No 261", ECDPM, 2019, https://ecdpm.org/wp-content/uploads/ Elections-Africa-Playing-Game-Bending-Rules-ECDPM-Discussion-Paper-261.pdf. 
RENAMO aveva totalizzato quasi il $32 \%$, mentre il resto dei voti si era disperso, come al solito, fra altri candidati di secondo piano, i cui partiti non riuscirono a eleggere alcun deputato in parlamento.

Il risultato fu soddisfacente, ma al di sotto delle aspettative: Guebuza aveva condotto una campagna elettorale di discontinuità rispetto a Chissano, accusato indirettamente di aver favorito il deixa-andar ("lascia andare", in riferimento alle diffuse pratiche di corruzione presenti nel paese), recuperando così la tradizione del primo e amatissimo presidente della repubblica, il socialista Samora Machel, scomparso in un incidente aereo il 19 ottobre del 198415. Tuttavia, durante il suo primo mandato, Guebuza, al di là della retorica politica, non soltanto non fece nulla per combattere la corruzione e le diseguaglianze interne, ma favorì la formazione di una nuova élite assai ristretta di fedeli del presidente, che dette ben presto l'assalto all'economia del paese, facendo del presidente della repubblica l'uomo più ricco del Mozambico e della figlia Valentina (poi assassinata dal marito nel dicembre del 2016) la sua equivalente al femminile, in pochissimo tempo ${ }^{16}$.

Il risultato più visibile di una simile governance poco inclusiva $\mathrm{e}$ altamente neo-patrimonialista fu che la rapida crescita economica non si diffuse fra gli strati più poveri della popolazione, come anche autori italiani hanno mostrato nei propri lavori ${ }^{17}$. Secondo dati della Banca Mondiale, se fino al 2008 la tendenza alla distribuzione della ricchezza fu impercettibilmente a favore del $50 \%$ più povero della popolazione, dal 2008 il trend si inverte rapidamente e decisamente, allargando il fossato fra la maggioranza indigente e una élite urbana ricca, numericamente sempre più ristretta ${ }^{18}$. In parallelo, le opportunità di accesso ai principali servizi (educazione, abitazione, sanità, ecc.) si differen-

15 Un buon ritratto, in chiave storico-filosofica, della figura di Samora Machel è presentato da S. Ngoenha, Ícone da Primeira República?, Maputo, Ndjira, 2004.

${ }^{16}$ Per avere una idea del vastissimo impero economico creato da Guebuza per sé e la propria famiglia, si veda l'articolo Império empresarial de Guebuza expande-se para aviação civil, in "A Verdade", 31/10/2016, https://macua.blogs.com/moambique_para todos /2016/10/imp\%C3\%A9rio-empresarial-de-guebuza-expande-se-para-avia\%C3\%A7\%C3\% A3o-civil.html.

${ }^{17}$ Cfr. C. TORNIMBENI, 50 anni di FRELIMO. Le diverse stagioni della democrazia in Mozambico, in “Afriche e Orienti”, n. 1-2, 2012, pp. 93 - 108; L. BussotTi, La democrazia in Mozambico: concettualizzazione e periodizzazione, in "La Critica Sociologica", Estate 2014, pp. 53-78

18 The World BAnK, Moçambique. Avaliação da pobreza, cit.. 
ziarono ulteriormente fra il 2008 e il 2016 non soltanto in rapporto all'appartenenza di classe, ma anche a livello territoriale (ossia etnico) e, all'interno di ciascun territorio, fra gli individui legati al sistema di potere del FRELIMO e coloro che ne erano esclusi.

Il primo campanello di allarme di una economia così diseguale fu lo scoppio di una violenta manifestazione popolare nel febbraio del 2008, nell'imminenza della campagna elettorale dell'anno successivo, concentrata fra Maputo e Matola, che mise in gravi difficoltà il governo del presidente Guebuza. Le misure assunte furono emergenziali e talmente superficiali che 1'1 e il 2 settembre del 2010 la manifestazione si ripetè, con una risposta ancora più violenta da parte del governo, e un bilancio di più di dieci morti fra $\mathrm{i}$ manifestanti ${ }^{19}$. Anche in questo caso, la comunità internazionale, da cui dipendeva circa la metà del bilancio dello stato mozambicano, non raccolse le denunce di organizzazioni nazionali dei diritti umani, come la Lega Mozambicana dei Diritti Umani con la sua leader, Alice Mabote, così come internazionali, fra cui Amnesty International e Human Rights Watch ${ }^{20}$.

Guebuza e il FRELIMO, forti del risultato del 2004, ma consapevoli della forza della RENAMO, soprattutto nel centro e nord del paese, organizzarono la macchina elettorale in tempo e con molta attenzione rivolta all'appuntamento del 2009. Ma, in quel preciso momento politico, ancor più della RENAMO, che era un nemico conosciuto e con cui l'élite del FRELIMO sapeva come rapportarsi, ciò che spaventava maggiormente era un rischio ancora poco noto: il Movimento Democratico del Mozambico (MDM), il nuovo partito fondato da Daviz Simango proprio nel 2009, in seguito a una clamorosa vittoria dello stesso Simango alle amministrative dell'anno prima. In quell'anno una lista civica improvvisata lo aveva eletto sindaco di Beira (la seconda città del Mozambico) per la seconda volta (nel 2003 era stato appoggiato dalla RENAMO), contro i "giganti" del FRELIMO e della RENAMO. Bisognava quindi affrontare il rischio, se non di una vittoria, almeno di un successo elettorale si-

${ }^{19} \mathrm{Su}$ questo si veda L. De Brito (ed.), Agora eles têm medo de nós, Maputo, IESE, 2017.

${ }^{20} \mathrm{Su}$ questi episodi e su altri tipi di violazione da parte della polizia mozambicana, cfr. L.A. NHAUELEQUe, Os direitos humanos na policia moçambicana, in "Debates Insumbissos", n. 8, 2020, pp. 62-91, in: https://periodicos.ufpe.br/revistas/debatesinsubmissos/article/view/243948. 
gnificativo dell'MDM, che lo avrebbe proiettato sullo scenario nazionale come la vera novità della politica mozambicana.

L'MDM evocava nella vecchia classe dirigente del FRELIMO reminiscenze poco piacevoli, che potevano far pensare a un contrappasso della storia mozambicana che si sarebbe realizzato attraverso la figura di Daviz Simango. Questi, infatti, era il figlio di Uria Simango, che fu vice-presidente del FRELIMO ai tempi di Eduardo Mondlane, poi ucciso barbaramente con la moglie Celina come "traditore della patria" nei campi di rieducazione (o meglio di concentramento) istituiti nel nord del paese dal governo socialista che allora monopolizzava la scena politica locale, in un anno ancora oggi imprecisato, ma probabilmente fra il 1980 e il $1985^{21}$.

L'obiettivo principale del FRELIMO per le elezioni del 2009 era, quindi, limitare il potenziale elettorale dell'MDM, cosa che fu fatta usando una delle armi preferite dal partito al potere: sfruttare le pieghe formali della legge (L. 29/2009) e la benevolenza degli organismi cosiddetti di controllo, in primo luogo la CNE e il suo presidente, il professor João Leopoldo da Costa, oltre alla consueta complicità, passività o rassegnazione dei partner internazionali, al fine di eliminare dalla competizione elettorale diversi candidati e partiti politici. Furono infatti esclusi totalmente 10 candidati su 29 per le elezioni presidenziali e legislative, mentre l'MDM, che era il vero obiettivo di tali misure, era stato escluso da 9 circoli elettorali su un totale di 14, togliendo quindi allo stesso qualsiasi possibilità di vittoria.

Gli osservatori internazionali, soprattutto l'Unione Europea, avevano tentato di segnalare come negative tali esclusioni22. Tuttavia, il modello di cooperazione internazionale a suo tempo concordato col Mozambico escludeva la possibilità di un intervento da parte dell'Unione Europea (come dei singoli paesi) nel processo elettorale. Motivi di "sovranità nazionale" invocati dal governo impedivano una collaborazione più stretta in questo delicato ambito, sebbene non lo impedissero quando si trattava di ricevere cospicui aiuti per le politiche di Budget Support e per gli altri mille rivoli di appoggi settoriali e umanitari23.

${ }^{21}$ Sulla vicenda di Uria Simango, cfr. B.L. Ncomo, Uria Simango: um homem, uma causa, Maputo, Novafrica.

22 M. Guente, Missão da Observação da União Europeia inconformada com a exclusão dos partidos pela CNE, in "Canalmoz", 02/11/2009.

${ }^{23}$ M. De Tollenaere, Apoio a Democratização a Moçambique pós-conflito, The 
La stampa privata locale aveva criticato i motivi formali alla base dell'epurazione elettorale che toccò anche l'MDM, con il professor João Leopoldo da Costa additato come principale colpevole e mero esecutore della volontà del partito al potere ${ }^{24}$. Ma anche questo non servì a far recedere il FRELIMO dal suo piano finalizzato a preparare una vittoria bulgara per il proprio candidato alla presidenza, Guebuza. Fu questo l'inizio dell'inversione della transizione democratica verso un modello autoritario, mediante l'uso partitico delle principali istituzioni della repubblica.

3. L'affermazione della transizione autoritaria: le elezioni del $2009 e$ la loro gestione

Il piano organizzato dal FRELIMO dette risultati ottimi. Il risultato finale delle elezioni fu infatti chiarissimo: Guebuza trionfò con il $75 \%$ dei consensi, la RENAMO fu ridotta a un modesto 16\% e l'MDM all' $8,6 \%$. Lo stesso risultato, con pochi decimi di differenza rispetto al candidato Guebuza, fu ottenuto dal FRELIMo per le elezioni legislative, fatto che assicurò al partito la maggioranza qualificata in parlamento, aprendo così la possibilità che Guebuza forzasse questo organismo legislativo a cambiare la costituzione per permettergli di effettuare un terzo mandato. Un tentativo, questo, che condizionò l'intero secondo mandato di Guebuza, come si vedrà in seguito.

I principali partiti di opposizione non ebbero molto spazio di manovra per proporre ricorsi tali da annullare il risultato elettorale. Dhlakama, lo storico presidente della RENAMO, scomparso il 3 maggio del 2018, non accettò i risultati, fece ricorso e lo perse, come era sempre accaduto nelle occasioni precedenti. Egli portò prove all' apparenza inconfutabili, tratte dalla stessa relazione del Consiglio Costituzionale sulle elezioni, come il caso simbolico di un seggio elettorale in cui erano iscritti 200 elettori, avevano votato in 50, e Guebuza aveva ottenuto1000 voti25. I dieci lea-

Hauge, Netherland Institute of International Relations, June 2006, in: www.clingendael.org.

${ }^{24}$ B. Nhamirre, CNE provaca terramoto político, in "Canalmoz", 08/09/2009, in: https://macua.blogs.com/moambique_para_todos/2009/09/cne-provoca-terramotopol\%C3\%ADtico.html.

${ }^{25}$ LUSA, Órgão moçambicano admite fraude em eleição, diz opositor, Lisboa, Agência Lusa, 30/12/2009, in: https://noticias.uol.com.br/ultnot/lusa/2009/12/30/ult611u83695.jhtm. 
der dei partiti di opposizione che non riuscirono a eleggere deputati chiesero anch'essi l'annullamento delle elezioni, ma queste richieste caddero nel vuoto.

Il risultato finale fu proclamato dal Consiglio Costituzionale e dal suo presidente, Luís Mondlane, soltanto il 28 dicembre dello stesso anno, con l'apertura di 229 processi contro 245 accusati di frodi comprovate, la più frequente delle quali consisteva nell'avere inserito nelle urne voti prestampati a favore di Guebuza e del FRELIMO ${ }^{26}$. Ma il risultato fu confermato, consegnando all'oblio osservazioni e critiche da parte della magistratura mozambicana.

L'Unione Europea, nonostante le proprie osservazioni pre-elettorali, stilò un rapporto finale positivo. Il voto, infatti, si era svolto nella calma e l'intero processo era stato ben organizzato; il quadro legislativo, nonostante qualche limite, fu considerato accettabile, mentre l'organizzazione logistica da parte dell'organismo preposto, lo STAE (Segretariato Tecnico dell'Amministrazione Elettorale) fu giudicata in modo eccellente; la campagna elettorale venne ritenuta come più pacifica del solito, con pieno rispetto per le pari opportunità di genere ${ }^{27}$. Altri organismi internazionali assunsero una posizione ancora più favorevole, in nome di un principio tacito: quello della non interferenza reciproca fra paesi africani, molti dei quali con situazioni peggiori dello stesso Mozambico quanto a trasparenza elettorale. L'Unione Africana, per esempio, definì il processo elettorale mozambicano del 2009 come "esemplare", mediante le dichiarazioni del proprio capo-missione, il vice-presidente angolano Roberto de Almeida, membro dell'MLPA, partito storicamente alleato del FRELIMO e, in quegli anni, al governo di un paese ancor meno democratico del Mozambico, dominato dalla figura di Eduardo dos Santos. Anche la delegazione della SADC (Southern Africa Development Community), l'aggregazione regionale degli stati dell'Africa Australe, sostenne che le elezioni erano state trasparenti e giuste ${ }^{28}$.

${ }^{26}$ B. NhAmirre, Validação da vitória da Frelimo e de Guebuza, in "Canalmoz", 29/12/2009, in https://macua.blogs.com/moambique_para_todos/2009/12/valida $\%$ C3\% A $7 \% \mathrm{C} 3 \%$ A3o-da-vit\%C3\%B3ria-da-frelimo-e-de-guebuza.html

27 European Parliament. Election Observation Delegation, Presidential, Parliamentary and Provincial Elections in Mozambique. Report by Cristian Dan Preda, Maputo, European Union in Mozambique, 2009, www.europarl.europa.eu .

${ }^{28}$ M. Guente, Missão da Observação, cit.. 
Una linea abbastanza simile sostenne anche Nuvunga, che negli anni successivi sarà assai critico, ma che, rispetto alle elezioni del 2009, espresse un giudizio positivo, affermando che erano state condotte in un clima di relativa pace, riconoscendo all'élite politica di aver saputo combinare competizione politica, cooperazione e stabilità ${ }^{29}$.

Sul fronte opposto, uno dei pochi osservatori internazionali ad assumere una posizione piuttosto critica fu Joseph Hanlon, che - in una analisi dettagliata - ritenne centrale il ruolo svolto dalla CNE nella tornata elettorale del 2009, in almeno tre aspetti fondamentali: la scarsa trasparenza, la poca flessibilità nei confronti dei reclami delle opposizioni, e la mancanza di puntualità nelle risposte, giocando largamente sulle ambiguità legislative ${ }^{30}$. Lo stesso Hanlon conclude che quelle elezioni furono caratterizzate da scarsa trasparenza classificandole quindi come poco credibili31.

Sarebbe riduttivo interpretare ciò che accade nel quadro politico mozambicano come esclusiva volontà, da parte di Guebuza, di restare al potere per un altro mandato. Tuttavia, in contesti africani di stati istituzionalmente fragili ma autoritari, non è raro riscontrare casi in cui il presidente della repubblica "prende in ostaggio" il paese. Altri esempi nella regione australe sono stati lo Zimbabwe con Mugabe, che cedette il potere ormai ultranovantenne nel 2017 dopo 37 anni di presidenza, in seguito a una sorta di colpo di stato pacifico che riavviò la normale dialettica democratica ${ }^{32}$; o l'Angola, per quasi quaranta anni dominata dalla figura di Eduardo dos Santos e famiglia, soprattutto della figlia Isabel ${ }^{33}$.

In Mozambico la propensione per soluzioni autoritarie sta scritta nella stessa costituzione. Questa, infatti, attribuisce al presidente della repubblica poteri enormi, non essendosi registrata, da questo punto di vista,

${ }^{29}$ A. Nuvunga, M.A.M. Salih, Mozambique's 2009 elections: framing democratic consolidation in contexto, in "Journal of African Elections", n. 1, 2010, pp. 34-53.

30 J. Hanlon, Post-Election Review of the Mozambique Elections of 2009, in https://www.open.ac.uk/technology/mozambique/sites/www.open.ac.uk.technology.mozambique/files/files/Mozambique\%202009\%20election\%20Hanlon\%20EISA-1.pdf.

31 J. HANLON, Frelimo landslide in tainted election in Mozambique, in "Review of African Policial Economy", n. 123, march 2010, pp. 92-95.

$32 \mathrm{BBC}$, Zimbabwe's Robert Mugabe resigns, ending 37-year rule, in "BBC News", 21/11/2017, in: https://www.bbc.com/news/world-africa-42071488.

33 VOA PORTUGUÊS, Luanda Leaks: o império de Isabel dos Santos através de empresas de fachada, 19/01/2020, in: https://www.voaportugues.com/a/luanda-leaks-o-imp\%C3\%A9 rio-de-isabel-dos-santos-atrav\%C3\%A9s-de-empresas-de-fachada/5252295.html. 
alcuna soluzione di continuità rispetto alla costituzione socialista del 1975. Gli Accordi Generali di Pace, per esempio, non hanno toccato questo cruciale aspetto, lasciando che l'approvazione della nuova costituzione del 1990 e poi la sua revisione del 2004 permettessero l'accumulazione di poteri nella figura del capo dello stato, senza una effettiva divisione degli stessi fra le varie istanze istituzionali. Ciò ha fatto in modo che fosse impossibile garantire elezioni libere e trasparenti. Un sistema che, durante il lungo periodo alla presidenza della repubblica di Chissano, ha in parte funzionato grazie alla capacità diplomatica dimostrata da questa figura politica, ma che ha assunto tutt'altro tono con l'elezione di Guebuza, soprattutto dopo la sua seconda elezione nel 2009.

Tuttavia la governance "liberale" di Chissano aveva portato a una quasi-sconfitta elettorale nel 1999, fatto che indusse il FRELIMO a ricercare fra le proprie fila una figura completamente diversa per la successione. Da qui la scelta di Armando Emílio Guebuza, fautore della "linea dura", ritenuta necessaria per riportare ordine nel paese e limitare il rischio di cedere il potere alla RENAMO.

Soprattutto nella sua seconda legislatura da presidente (2009-2014), dopo il trionfo personale alle elezioni, Guebuza prese alla lettera tale compito, accentrando sempre di più tutte le principali decisioni da assumere per il paese, e identificando nell'eccesso di democrazia e di libertà il vero pericolo di una eventuale sconfitta elettorale per mano della RENAMO (o, dal 2009, anche dell'MDM).

A Guebuza non restava che "normalizzare" il paese secondo i suoi propositi, avendo come primo obiettivo la sua stessa rielezione o, in subordine, la successione della moglie, Maria da Luz Guebuza, particolarmente attiva nella vita sociale a anche politica nel ruolo di Primeira Dama. Prima, però, doveva passare per un analogo processo all'interno del partito, escludendo gli oppositori, soprattutto e paradossalmente il gruppo di vecchi frelimisti più legati alla memoria di Samora Machel, come Oscar Monteiro, Jorge Rebelo e altri di quella generazione, e rimodellando il Comitato Centrale e il Comitato Politico, i due organismi decisivi per le principali scelte politiche del paese, a partire dall'indicazione del candidato a presidente della repubblica, inserendovi suoi fedeli. Tale processo avvenne nel settembre del 2012, al X Congresso del Frelimo svoltosi a Pemba, di cui si dirà più avanti.

Dal punto di vista della governance, Guebuza approfittò dell'eccellente risultato elettorale per dare maggiore dinamismo all'azione 
amministrativa, puntando soprattutto su tutto ciò che desse visibilità, anche a livello internazionale. L'idea che il presidente intendeva trasmettere era quella di un esecutivo efficiente, al quale si poteva anche perdonare una gestione poco democratica dello stato. Così, nel 2011 il Mozambico ospitò, per la prima volta, i giochi pan-africani, una sorta di olimpiadi continentali, ristrutturando o costruendo ex-novo stadi e altri impianti sportivi. I giochi rappresentarono un successo di notevoli proporzioni per Guebuza, che ricevette le congratulazioni di tutta la comunità internazionale ${ }^{34}$. In parallelo, il governo mozambicano completò a tempo di record alcune grandi opere infrastrutturali, che Guebuza volle, indipendentemente dal loro effettivo impatto socio-economico e finanziario sulle casse dello stato. Ad esempio, fu completato il ponte sul fiume Zambeze (già nel 2009) che unisce il centro col nord del paese (province di Sofala e Zambezia), non a caso denominato "Ponte Armando Emílio Guebuza", così come il ponte che collega il Mozambico alla Tanzania sul fiume Rovuma (nel 2010), antico sogno dei due presidenti Machel e Nyerere, ma che soltanto Guebuza riuscì a realizzare al costo di 35 milioni di euro avvalendosi di una impresa cinese. Infine, fu con Guebuza che si dette inizio ai lavori del più grande ponte sospeso di tutta l'Africa, che collega la città di Maputo con Katembe, l'altro lato della città, separati da una lingua di Oceano Indiano. Inaugurato dal successore di Guebuza, Nyusi, nel 2018, questa infrastruttura costò 276 milioni di dollari, di cui il 95\% finanziati dalla cinese Exim Bank e il 5\% dal bilancio dello stato. I lavori furono realizzati dalla China Road and Bridge Corporation, e il processo di reinsediamento delle popolazioni toccate dall'opera fu difficile e violento. Se i cinesi diventarono i partner privilegiati sotto Guebuza, l'altro asse prioritario fu identificato nei grandi investimenti, o mega-progetti, tutti forieri di grandi polemiche e opposizioni popolari, viste le modalità autoritarie con cui tali investimenti furono realizzati 35 .

${ }^{34} \mathrm{Su}$ questo cfr. L. BussotTi, Os X jogos africanos de Maputo: o desporto como veículo da promoção da imagem nacional dum país na imprensa local, in A. NASCIMENTO, M. Bittencourt, N. Domingos, V.A. De Melo, (eds.), Esporte e Lazer na África: Novos Olhares, Rio de Janeiro, 7Letras, 2013, pp. 241-266.

35 Sulle proteste sociali contro i mega-progetti in Mozambico, cfr. R. MutzenBerG, Protestos sociais em Moçambique: uma agenda de pesquisa, in "Civitas", n. 1, 2014, pp. 137-153, https://revistaseletronicas.pucrs.br/ojs/index.php/civitas/article/view/ $16196 / 10965$ 
Contemporaneamente a queste gigantesche opere pubbliche, le ricchezze personali di Guebuza e famiglia crescevano a dismisura, grazie a reti diffuse di corruzione, che portarono il paese a un peggioramento repentino sia rispetto agli indici di corruzione internazionali che agli indici di diffusione della povertà ${ }^{36}$.

Per garantire il buon funzionamento di una macchina tanto complessa, Guebuza doveva imporre il modello dell'uomo solo al comando. Gli strumenti, anche nella prospettiva di una sua terza elezione a capo dello stato che avrebbe dovuto passare per forza da una riforma costituzionale approvata dal parlamento, furono essenzialmente tre: in primo luogo, occorreva limitare la libertà di critica dei giornali privati che non erano certo favorevoli alla sua strategia politica; in secondo luogo, occorreva preparare adeguatamente il proprio partito a questo percorso; infine, era necessario annullare le residue resistenze della RENAMO.

Quanto al primo obiettivo, le principali azioni furono due: da un lato, Guebuza promosse una sorta di task-force di intellettuali di regime, coordinati dal portavoce del proprio governo, Edson Macuácua, un brillante giovane emergente, che avrebbero dovuto organizzare gli interventi negli organi di informazione e nelle reti sociali (oltre che, in collaborazione col sise, l'intelligence locale, nelle scuole e soprattutto nelle università) al fine di condizionare l'opinione pubblica nazionale. Il gruppo assunse il nome di G40, ed ebbe un ruolo significativo nell'inquinare il dibattito politico con azioni programmate e coordinate. Il controllo sulla stampa nazionale pubblica, dalla principale radio del paese, RM, alla televisione più seguita, la TVM, dall'unico quotidiano di diffusione nazionale, Notícias, fino al settimanale Domingos, divenne inoltre totale. La nuova linea impose la sostituzione di direttori scomodi, che cercavano di esprimere critiche (anche se costruttive) all'azione di governo, come accadde a Rogério Sitoe, brillante giornalista e direttore di Notícias, deposto da un giorno all'altro e sostituito da un personaggio del tutto incompetente, ma fedelissimo di Guebuza. Sul fronte della stampa privata, fu dato l'ordine di eliminare qualsiasi tipo

36 Vedasi su questo F. BıcHO, Moçambique é um caso problemático no que toca à corrupção, diz pesquisa, in "DW", 09/07/2013, https://www.dw.com/pt-002/mo\%C3\%A7ambique- $\% \mathrm{C} 3 \% \mathrm{~A} 9$-um-caso-problem $\% \mathrm{C} 3 \% \mathrm{~A} 1$ tico-no-que-toca- $\% \mathrm{C} 3 \% \mathrm{~A} 0$-corrup $\% \mathrm{C} 3 \% \mathrm{~A} 7$ \%C3\%A3o-diz-pesquisa/a-16939542. 
di pubblicità da parte di imprese pubbliche a giornali come Canal de Moçambique, mentre misure speciali furono prese contro il gruppo soICO, il più importante gruppo nazionale privato nel settore dei mezzi di comunicazione di massa, che fu costretto a sostituire Jeremias Langa, direttore dell'informazione, per i suoi programmi televisivi di opinione, ritenuti eccessivamente critici verso il governo ${ }^{37}$.

Una volta normalizzata la stampa (almeno così Guebuza credeva), fu la volta del partito. Al X Congresso del FRELIMO svoltosi a Pemba nel settembre del 2012, Guebuza riformò tutti i principali apparati decisionali del partito, spostando a proprio favore gli equilibri interni, introducendo un principio nuovo per questa formazione politica: la possibilità di scindere la figura del presidente della repubblica da quella del presidente del FRELIMO, nel caso in cui tutti i suoi tentativi di rielezione fossero stati sconfitti ${ }^{38}$. Guebuza era adesso pronto per scatenare una nuova guerra contro la RENAMO e il suo leader, Dhlakama, facendola passare per un conflitto provocato non dal suo governo, ma dallo stesso Dhlakama, in sofferenza a causa delle frodi registrate nelle ultime elezioni del 2009. Tale opzione doveva servire, a breve termine, per rimandare le elezioni del 2014, avendo così il tempo - a medio termine - per obbligare il parlamento a quella riforma costituzionale che gli avrebbe permesso di essere candidato per effettuare il suo terzo mandato da presidente della repubblica, completando così il suo disegno autoritario. Questo tentativo di Guebuza si iscrive all'interno di un quadro politico che, come detto inizialmente, è figlio di sfiducia e violenza simbolica fra i due partiti che da sempre hanno monopolizzato lo scenario elettorale mozambicano, nonché di questioni mai risolte, provenienti dagli Accordi Generali di Pace, ma che Guebuza intendeva affrontare in modo definitivo.

La RENAMO - che aveva sempre dalla sua l'arma di un esercito da usare eventualmente per convincere il FRELIMO a mutamenti ritenuti necessari, in nome del completamento della transizione democratica stava ponendo, dopo le elezioni del 2009, questioni di fondo legate a

${ }^{37}$ Cfr. L. Bussotti, Media Freedom and the Transition Era in Mozambique 19992000, in L. Bussotti, M. De Barros, T. Grätz, (eds.), Media Freedom and Right to Information in Africa, Lisbon, ISCTE, in https://books.openedition.org/cei/145.

${ }^{38} \mathrm{Cfr}$. DW, X Congresso da Frelimo evidencia queda de braço interna ao partido, in "DW", 24/09/2012, https://www.dw.com/pt-002/x-congresso-da-frelimo-evidencia-quedade-bra\%C3\%A7o-interna-do-partido/a-16258219. 
tre aspetti centrali. Il primo si riferiva alla necessità di implementare meccanismi in grado di limitare al massimo le frodi elettorali da parte del partito-stato FRELIMO. Le proposte si diressero verso una nuova legge elettorale, la riforma della CNE e un pacchetto legislativo in grado di far eleggere i governatori provinciali in modo diretto, superando così il meccanismo delle nomine presidenziali. Il secondo riguardava il completamento del processo di smobilitazione e reintegro dei propri militari ancora armati, secondo quanto stabilito dagli Accordi. Infine, il terzo punto era il più ampio e forse ambizioso, e riguardava la richiesta di "defrelimizzare" lo Stato, distribuendo in modo più equo le ricchezze del paese. Incontri infiniti furono effettuati presso il Centro di Conferenze Joaquim Chissano a Maputo fra le due parti, ma questi non portarono ad alcuna soluzione, anche perché il capo-delegazione del governo, José Pacheco, al tempo ministro dell'agricoltura, era considerato un fedelissimo di Guebuza e non aveva alcuna intenzione di accettare le richieste della RENAMO.

Proprio durante i negoziati, ad aprile 2013, Guebuza dette l'ordine di avviare la "soluzione finale", ossia il definitivo annientamento della RENAMO e delle sue basi militari. Santunjira, località presso la Serra di Gorongosa dove si trovava Dhlakama coi suoi uomini, venne accerchiata dai militari dell'esercito in assetto di guerra, rendendo in pratica impossibile, per il leader della RENAMO, uscire da quella località. Le poche volte che riuscì a farlo fu oggetto di due attentati da parte di militari dell'esercito regolare, uno a Manica, l'altro nella sua casa a Beira, ma entrambi fallirono. La polizia di stato procedette poi all'arresto di 15 uomini armati della RENAMO anche a Manica, mentre erano di scorta armata al segretario generale del partito, Manuel Bissopo, che si trovava in una località turistica a Chimoio, rompendo così quell'implicita tolleranza reciproca, per la quale la RENAMO manteneva l'esercito, che usava tuttavia soltanto come arma di pressione per ottenere le riforme istituzionali dal FRELIMO ${ }^{39}$. La situazione si aggravò nel 2013 e 2014, con una escalation che portò presto a un nuovo conflitto armato nel centro del paese.

${ }^{39}$ L. MATIAS, Negociações entre RENAMO e Governo moçambicano marcadas por finca-pé, in "Dw", 02/05/2013, https://www.dw.com/pt-002/negocia\%C3\%A7\%C3\%B5 esentre-renamo-e-governo-mo $\% \mathrm{C} 3 \% \mathrm{~A} 7 \mathrm{ambicano}$-marcadas-por-finca-p $\% \mathrm{C} 3 \% \mathrm{~A} 9 / \mathrm{a}$ 16786583 . 
Il 2014 fu l'anno in cui una nuova guerra fra le due parti iniziò per così dire ufficialmente, con tanto di mediatori internazionali, capeggiati dall'Unione Europea e dall'italiano Mario Raffaelli, uno dei diplomatici (al tempo vice-ministro degli affari esteri di Roma) che riuscirono a concludere il primo, lungo conflitto fra le parti con gli Accordi Generali di Pace del 1992. Il 5 settembre 2014 fu sottoscritto un accordo di cessazione delle ostilità, firmato da Guebuza e Dhlakama, così da permettere la celebrazione delle elezioni a fine 2014, come da calendario $^{40}$. Queste si svolsero in un clima di altissima tensione ma la terza candidatura di Guebuza non potè realizzarsi dal momento che il parlamento non arrivò nemmeno a discutere la riforma costituzionale che sarebbe stata necessaria per effettuare tale operazione.

\section{Il consolidamento del modello autoritario: le elezioni del 2014 e la nuova governance Makonde}

All'interno del FRELIMO la decisione sulla successione di Guebuza fu molto sofferta anche a causa dei suoi continui tentativi di succedere a se stesso, nonostante gli impedimenti costituzionali. Il partito, infatti, aveva scelto una propria linea: coerentemente con i patti da tempo stabiliti, dopo tre presidenti del Sud, tutti Ronga, doveva essere la volta di un Makonde, in ossequio alla salda e antica alleanza fra queste due etnie. La persona indicata era il generale Alberto Chipande, leader dei Makonde, che però, soprattutto per motivi anagrafici, rinunciò, indicando un proprio famigliare, l'allora ministro della difesa Filipe Nyusi, quale candidato per la presidenza. Dall'altra parte, l'ex-prima ministra, l'economista Luísa Diogo, di Tete, provò a contrapporsi alla candidatura di Nyusi, che però vinse le primarie interne, appoggiato, alla fine, anche da Guebuza e dai suoi fedeli, che considerarono Nyusi come il male minore.

Nyusi, infatti, era stato direttamente coinvolto, come ministro della difesa - anche se al tempo la questione era conosciuta esclusivamente all'interno della ristretta cerchia dei vertici del FRELIMO - nello scandalo

40 Un'ottima ricostruzione di questi fatti è proposta da A. VINES, As perspectivas de um acordo sustentável entre as Elites em Moçambique, London, Chattam House, 2019, in https://www.chathamhouse.org/sites/default/files/2019-09-04-ElitePerspectivesMozambique-Portuguese.pdf 
del debito pubblico occulto, deflagrato nel 2016. Una operazione, questa, ancora sotto le lenti della giustizia internazionale (tribunali di New York e Londra) e nazionale, la cui conseguenza più grave è stata la sospensione del Budget Support da parte dei partner occidentali, e la loro totale perdita di fiducia nei confronti del Mozambico. E, in chiave interna, la conseguenza fu il crollo dell'immagine di Guebuza, dentro e fuori il partito, con l'arresto di uno dei suoi figliti.

Le elezioni del 2014 furono ancora peggiori, quanto al loro svolgimento, rispetto a quelle del 2009, oltre che più incerte. Il clima di tensione politico-militare presente nel paese accentuò la polarizzazione fra FRELIMO e RENAMO, e fra i rispettivi candidati, lasciando ai margini l'MDM, che rimase schiacciato fra i due vecchi contendenti, perdendo molto del proprio appeal iniziale. I risultati finali dettero la vittoria a Nyusi, che si attestò al 57\%, quasi 20 punti in meno rispetto alla seconda elezione di Guebuza, seguito da Dhlakama col 36,6\% e da Simango col 6,3\%. In parlamento il FRELIMO perdette 47 seggi a favore soprattutto della RENAMO $(+31)$ e, in parte, dell'MDM (+9 rispetto al 2009, con un totale di 17 deputati).

La RENAMO conquistò una larga maggioranza nelle elezioni legislative (e provinciali) in Zambezia e Sofala, e riuscì a vincere, anche se meno nettamente, in tutte le altre province centro-settenrionali del paese, con l'eccezione di Cabo Delgado. Nonostante tale risultato, la vittoria fu attribuita al FRELIMO e al suo candidato, Nyusi, e tutti i ricorsi fatti (23 in totale) proposti dalla RENAMO e dall'MDM furono respinti per ragioni formali (essenzialmente perché presentati dopo le 48 ore consentite dalla legge). L'unico ricorso fatto dal FRELIMO, a Tsanago, in provincia di Tete, fu invece accolto, il che provocò la ripetizione delle elezioni, poiché i membri della RENAMO avevano distrutto le installazioni dove avrebbe dovuto svolgersi la votazione ${ }^{42}$.

I principali partner internazionali sottolinearono sì maggiori irregolarità e brogli rispetto alle precedenti elezioni del 2009, ma conti-

${ }^{41} \mathrm{Cfr}$, su questo: A. WiLliams \& J. IsAKSEn, Corruption and state-backed debts in Mozambique, in U4 Issue, November 2016, No 6, https://www.cmi.no/publications/file/6024corruption-and-state-backed-debts-in-mozambique.pdf

42 J. Hanlon, Eleições Nacionais 2014, in "Boletim sobre o Processo Político em Moçambique", N. EN 75, 02/11/2014, https://www.open.ac.uk/technology/mozambique/ sites/www.open.ac.uk.technology.mozambique/files/files/Elei\%C3\%A7\%C3\%B5es_Nacionais_75-2deNovembro-2015_comentarios_dados.pdf 
nuarono a esprimere un giudizio positivo sul processo elettorale mozambicano del 2014. Ė questa la posizione sia dell'Unione Europea ${ }^{43}$ che della Fondazione Carter, la quale volle sottolineare il clima di relativa tranquillità in cui si erano svolte le elezioni, piuttosto che le frodi denunciate, a cui non dette, in pratica, alcun ascolto ${ }^{44}$.

Tuttavia, alcuni osservatori che avevano espresso un giudizio positivo sulle elezioni del 2009, in occasione di quelle del 2014 assunsero un atteggiamento assai più critico. Adriano Nuvunga, per esempio, descrisse tali elezioni come caratterizzate da frodi e da un atteggiamento di segretezza da parte della $\mathrm{CNE}$; accentuò tuttavia anche la scarsa perizia, da parte della RENAMO, nell'usare gli strumenti legislativi che la legge metteva a disposizione per proporre ricorsi puntuali e circostanziati, concludendo come l'intero sistema politico-elettorale mozambicano fosse inadeguato e dovesse quindi essere radicalmente riformato ${ }^{45}$. Sulla stessa linea si collocò anche Joseph Hanlon, coerentemente con quanto da lui già segnalato in occasione delle elezioni del 200946.

La vittoria della RENAMO in gran parte del paese, oltre a non attribuire la vittoria finale a Dhlakama, non fece cambiare il vecchio obiettivo del FRELIMO di non condividere il potere con l'antico rivale, neanche a livello provinciale. I governatori, infatti, furono nominati dal presidente della repubblica, secondo la normativa dell'epoca, e Nyusi li scelse tutti fra i membri del FRELIMO, ignorando il responso delle urne. Si aprì, allora, un lungo periodo di altissima tensione nel paese, con Dhlakama a incendiare le folle, proponendo prima un "governo di gestione" (ossia, di unità nazionale), poi una riforma federalista dello Stato, e infine rivendicando la nomina di governatori provenienti dalla RENAMO in quelle province in cui il suo partito aveva ottenuto la maggioranza. Fu anche firmata una

43 European Union - Election Observation Mission, Mozambique. Final Report. General Elections, 15 October 2014, Maputo, 2014, in https://www.eods.eu/library/ eueom_mozambique_2014_finalreport_en.pdf

${ }^{44}$ The CARTer CEnTER, Preliminary Statement, Maputo, 2014, in: https://www.cartercenter.org/resources/pdfs/news/pr/Mozambique-prelim-10-17-14.pdf

45 A. NuvUNGA, Mozambique's 2014 elections: a repeat of misconduct, political tension and Frelimo dominance, in "Journal of African Elections", Vol. 16, No 2, pp. 71-94, 2017

46 J. Hanlon, Eleições Nacionais 2014, in "Boletim sobre o Processo Político em Moçambique", N. EN 75, 02/11/2014, https://www.open.ac.uk/technology/mozambique /sites/www.open.ac.uk.technology.mozambique/files/files/Elei\%C3\%A7\%C3\%B5es_Nacionais_75-2deNovembro-2015_comentarios_dados.pdf 
sorta di accordo in questo senso il 17 agosto del 2016, su sollecitazione di Raffaelli, ma lo stesso venne immediatamente smentito, alla televisione pubblica TVM, dal mediatore governativo, il generale Jacinto Veloso, che nel frattempo aveva sostituito Pacheco, chiarendo che Nyusi mai avrebbe nominato governatori appartenenti alla RENAMO ${ }^{47}$.

La questione della condivisione del potere, seppure a livello locale, come forma di risarcimento per le frodi elettorali che si verificarono anche nelle elezioni del 2014 non trovò, quindi, soluzione positiva neanche attraverso l'accordo del 2016. Un accordo che era costato la vita a varie persone, a partire da quella del giurista franco-mozambicano Jilles Cistac, professore presso l'Università Eduardo Mondlane, il quale nel marzo del 2015 fu brutalmente assassinato in pieno centro di Maputo da una squadra di sicari, probabilmente per avere espresso un parere positivo sulle richieste di federalismo della RENAMO. La stessa sorte toccò a vari membri della RENAMO nel corso degli anni, fra i quali Jeremias Pondeca, anch'egli ucciso a sangue freddo a Maputo, mentre praticava il jogging mattutino, nell'ottobre del 2016, mentre una ondata di intimidazioni colpì anche giornalisti e giornali indipendenti, come Paulo Machava assassinato il 28 agosto del 2015, Ericino de Salema, rapito e al quale vennero spezzate entrambe le gambe da un gruppo di sicari professionisti nel marzo del 2018, Matias Guente, editore del settimanale Canal de Moçambique, sfuggito a un rapimento per miracolo il 31 dicembre del 2019 mentre - ancora in agosto del 2020 - la sede di questo giornale venne completamente incendiata.

Il progetto di normalizzazione iniziato da Guebuza continuava quindi con la nuova governance Makonde, la quale pare avere rafforzato - anche secondo testimonianze dirette pubbliche - l'uso degli squadroni della morte, al fine di mettere a tacere le voci critiche ${ }^{48}$.

Nonostante tale clima, la guerra fra governo e RENAMO cessò quando Dhlakama si decise a dichiarare, in rapida successione e unilateralmente,

${ }^{47} \mathrm{Cfr}$, in rapida successione, la notizia dell'accordo: W. MAPOTE, Moçambique: Nyusi nomenará governadores indicados pela Renamo, in "Voaportugues", 17/08/2016, https://www.voaportugues.com/a/mocambique-nyusi-nomeara-governadores-indicados-renamo/3468799.html; e l'immediata smentita: L. MATIAS, Governo moçambicano desmente nomeação de governadores provisórios da Renamo, in "DW", 17/08/2016, https:// www.dw.com/pt-002/governo-mo\%C3\%A7ambicano-desmente-nomea $\% \mathrm{C} 3 \% \mathrm{~A} 7 \%$ C3\%A3o-de-governadores-provis\%C3\%B3rios-da-renamo/a-19480852

${ }^{48} \mathrm{Cfr}$. L'intervista a uno dei membri di questi squadroni della morte governativi, dal 
una serie di tregue, che misero fine al conflitto armato nel centro del paese nel dicembre del 2016. Ciò ha anche permesso di trovare un accordo sulle riforme costituzionali ed elettorali durante il 2018, che si sono sostanziate nella L. 1/2018 (Legge di Revisione Puntuale della Costituzione), con la quale si sono svolte le elezioni amministrative nello stesso anno, e quelle provinciali del 2019. Infine, la firma dei terzi accordi di pace, siglati fra Nyusi e il nuovo leader della RENAMO, Ossufo Momade, succeduto a Dhlakama, è giunta l'1 agosto del $2019^{49}$.

La morte di Dhlakama segna una tappa decisiva nei rapporti fra FRELIMO e RENAMO: innanzitutto, gli accordi firmati sono ben lontani da quanto le due delegazioni avevano in precedenza concordato, lasciando irrisolti aspetti essenziali di cui si dirà fra poco; in secondo luogo tale evento segna una grave scissione all'interno della RENAMO, con la formazione di una Giunta Militare Autonoma guidata dal generale Nhongo, che non riconosce né gli accordi firmati da Nyusi e Momade, né la nuova leadership di quest'ultimo, proseguendo un conflitto a bassa intensità nel centro del paese. Conflitto che, di fatto, è terminato l'11 ottobre del 2021 con l'uccisione da parte dell'esercito mozambicano di Nhongo.

Gli accordi definitivi di pace constano di due grandi parti: una politico-istituzionale, l'altra relativa alle questioni militari. In entrambi i casi il FRELIMO ha limitato al massimo le richieste della RENAMO, di fatto inglobandola definitivamente nel proprio sistema di potere, e privandola anche dell'unica, vera arma di pressione, costituita dall'avere un esercito a disposizione con cui "trattare" col partito-stato.

In particolare, la parte politica degli accordi ha introdotto l'elezione dei governatori provinciali, in precedenza di nomina presidenziale. Tuttavia, la modalità di elezione è indiretta, a differenza di quanto le due delegazioni avevano concordato sul modello dell'elezione diretta dei

titolo Há esquadrões da morte para abater opositores, revela agente da Polícia da República de Moçambique, in "A Verdade", 11/03/2016, https://macua.blogs.com/moambique_para_todos $/ 2020 / 08 / \mathrm{h} \% \mathrm{C} 3 \% \mathrm{~A} 1$-esquadr $\% \mathrm{C} 3 \% \mathrm{~B} 5$ es-de-morte-para-abater-opositores-revela-agente-da-pol $\% \mathrm{C} 3 \% \mathrm{ADcia}$-da-rep $\% \mathrm{C} 3 \% \mathrm{BAblica}$-de-mo $\% \mathrm{C} 3 \% \mathrm{~A} 7$ ambiquerepet i\%C3\%A7\%C3\%A3o.html. Cfr. anche AMnESTy InTERnACIONAL, Virar a página!, London, 2019, https://www.amnesty.org/download/Documents/AFR4110192019 PORTUGUESE.PDF

49 A. Sebastiano, Moçambique: Nyusi e Momade assinam acorrdo de paz na Gorongosa, in "Dw", 01/08/2019, https://www.dw.com/pt-002/mo\%C3\%A7 ambique-nyusi-emomade-assinam-acordo-de-paz-na-gorongosa/a-49845768 
sindaci. E, soprattutto, il FRELIMO - nella logica di massima riduzione del rischio - ha introdotto una figura completamente nuova nell'ordinamento mozambicano, il Segretario di Stato Provinciale. Tale figura rappresenta il potere centrale nelle province, ha maggiori prerogative rispetto al governatore eletto dai cittadini, anche se indirettamente, ed è nominato dal presidente della repubblica. Inoltre, l'adeguamento del sistema elettorale da diretto a indiretto ha toccato anche i sindaci che, dalle elezioni amministrative del 2018 , vengono eletti non più direttamente, ma passando per il consiglio comunale.

Per quel che riguarda la parte relativa alla difesa, ossia alla smobilitazione e al disarmo degli uomini della RENAMO, la situazione è ancora peggiore: gli ufficiali di grado più elevato provenienti da questo partito sono stati collocati in pensione (come nel caso di Elias Dhlakama, fratello dell'ex leader Afonso Dhlakama) o destinati a ruoli marginali. La sicurezza dei fuoriusciti dalla RENAMO non è stata garantita, dato che questi sono spesso stati oggetto di violenza da parte dei militari governativi, come Nhongo ha più volte denunciato; l'integrazione di membri della RENAMO nel SISE (il servizio di intelligence) non si è verificato, dato che questi sono stati assegnati esclusivamente a esercito e polizia; infine, gli attacchi alle basi della Giunta Militare di Nhongo sono continuati, rispondendo a interessi convergenti della RENAMO di Momade e del partito-Stato, con la sola protezione del mediatore delle Nazioni Unite, l'ambasciatore svizzero Manzoni ${ }^{50}$ il cui intervento, tuttavia, non ha potuto risparmiare la vita a Nhongo.

Contestualmente al definitivo depotenziamento della RENAMO, il gruppo Makonde egemone ha proceduto, per la prima volta in Mozambico in modo esplicito, a una tribalizzazione (appunto in senso Makonde) dell'apparato dello Stato, distinguendosi in questo da quanto fatto da Guebuza. Dal modello autoritario di tipo familistico si è così passati a uno di carattere etnico, con conseguenze nefaste per tutto il paese.

La governance Makonde guidata da Nyusi ha, come prima misura, imposto il proprio controllo sui settori considerati nevralgici, quelli legati alla difesa e alla sicurezza. Al ministero della difesa fu nominato Salvador Mtumuke, zio di Nyusi, a capo dei servizi segreti il temutis-

${ }^{50} \mathrm{Cfr}$. L. CumBe, Governo continua a enganar Renamo no processo DDR, in "Zambeze", https://zambeze.info/?p=7911, 22/03/2021. 
simo Lagos Lidimo, anch'egli Makonde, nominato a gennaio del 2017, ma che dovette rinunciare pochi mesi dopo a causa di una malattia cardiaca, mentre a comandante generale della polizia fu indicato il giovane Bernardino Rafael, Makonde e familiare di Chipande, nell' ottobre del 2017; altri grandi generali della stessa etnia furono liberi di dedicarsi ai loro affari, non senza numerosi problemi di ordine per così dire sociale, con gravissime violazione dei diritti umani dei lavoratori ${ }^{51}$. D'altro canto, il generale Chipande ha avuto la possibilità di accumulare notevoli ricchezze grazie ai suoi interessi nel settore del trasporto marittimo e soprattutto del gas, dato che la sua società, la Profin Consulting, è la promotrice del gasdotto che da Cabo Delgado dovrebbe raggiungere il Sud Africa, con un investimento di circa 6 miliardi di dollari ${ }^{52}$.

La Makondizzazione dello Stato non poteva non passare anche per un processo analogo nel partito, che richiedeva che Guebuza ne lasciasse la presidenza. Ciò avvenne piuttosto presto, in una riunione politica ad alta tensione del partito, nel marzo del 2015. Le voci dello scandalo del debito pubblico occulto circolavano già negli ambienti del FRELIMO con molta insistenza, e Guebuza, insieme a uno dei figli (poi arrestato) ne era stato uno degli artefici. Questa situazione di oggettiva debolezza fece sì che i Makonde, con alleanze interne con gli storici antagonisti di Guebuza, avessero buon gioco, avendo così via libera al loro progetto di accaparramento del potere politico ed economico del paese ${ }^{53}$. Qualcuno ha addirittura parlato di un vero e proprio sistema mafioso messo in piedi dal FRELIMO, e in particolare dall'élite Makonde: FRELIMO, governo e business sarebbero, secondo questo importante studioso, la stessa cosa, confondendosi e sovrapponendosi ${ }^{54}$.

${ }^{51}$ LUSA, Gemsfield aceita pagar 7,25 milhões em indemnizações por abusos dos direitos humanos em Moçambique, 29/01/2019, in https://www.rtp.pt/noticias/mundo/gemfields-aceita-pagar-725-milhoes-em-indemnizacoes-por-abusos-dos-direitos-humanos-emmocambique n1125758.

52 J. HANLON \& J. AHLBACK, Chipande family behind gas pipeline plan, in "Mozambique News Reports and Clippings", No. 313, 14/03/2016, https://www.acismoz.com/wpcontent/uploads/2017/06/313\%20News\%20Reports\%20and\%20Clippings.pdf.

53 LUSA, O presidente da Frelimo, Armando Guebuza, demitiu-se este domingo da liderança do partido no poder em Moçambique. Horas depois, Filipe Nyusi era eleito para o cargo, in "Expresso", 29/03/2015, https://expresso.pt/internacional/guebuza-demite-seda-lideranca-da-frelimo $=\mathrm{f} 917596$.

54 J. Hanlon, Turning Mozambique into a Mafia, Resource-Cursed State, in "The Round Table. The Commonwealth Journal of International Affairs", June 2021. 
Per garantirsi la continuità del loro progetto, i Makonde dovevano dimostrare che il FRELIMO era uscito rafforzato dalla loro esperienza di governo. Col pacchetto legislativo approvato, si svolsero così le elezioni amministrative del 2018, e successivamente le presidenziali, legislative e provinciali del 2019. La critica specializzata, che aveva ormai compreso come il Mozambico non potesse più essere considerato un caso di successo, lesse questa doppia tornata elettorale come la peggiore della breve storia elettorale del paese: nel 2018 l'opposizione conquistò nove comuni, fra cui città importanti come Beira (a Simango dell'MDM), Nampula, Quelimane e l'antica capitale Ilha de Moçambique, tutte alla RENAMO, che sfiorò anche una storica impresa a Matola e perfino a Maputo, dove il conteggio dei voti fu macchiato da gravissime irregolarità, peraltro sottolineate dalla stessa società civile mozambicana ${ }^{55}$.

Visti i risultati non soddisfacenti delle amministrative del 2018, Nyusi e il suo governo prepararono al meglio, per così dire, le successive elezioni generali del 2019. La morte di Dhlakama, nel maggio del 2018, aveva spianato la strada all'ennesima vittoria del FRELIMO, che doveva confrontarsi nuovamente con Simango dell'MDM, ormai ampiamente depotenziato, e con Momade della RENAMO, un leader contestato e poco carismatico. Le elezioni si svolsero, poi, in un clima di guerra nel nord e nel centro del paese, limitando quindi molto l'effettiva possibilità, per molti mozambicani, di recarsi alle urne. Nyusi ottenne il $73 \%$ dei voti validi, Momade quasi il 22\% e Simango il 4,3\%. Il FRELIMO vinse in tutte le circoscrizioni elettorali del paese e in tutte le province, non lasciando che le briciole alle opposizioni. I meccanismi di frode generalizzata furono accompagnati anche da scontri e attentati, fra questi l'uccisione di un rappresentante del tavolo elettorale della società civile nella provincia di Gaza, Anastácio Matavele, i cui assassini sono stati identificati in 6 poliziotti dei reparti speciali, già condannati individualmente, ma con 1'assoluzione piena dello Stato mozambicano da qualsiasi co-responsabilità sull'accaduto ${ }^{56}$. Questi

55 Votar Moçambique, Posicionamento sobre as eleições autárquicas de 10 de Outubro de 2018, Maputo, 17/10/2018, in https://cipmoz.org/wp-content/uploads/2018/10/Posicionamento-Votar-Moc\%CC\%A7ambique.processo-eleitoral_-Revisto-3.pdf.

${ }^{56}$ C. Matsinhe, Caso Anastácio Matavele: Seis polícias condenados a prisão, in "DW", 18/06/2020, https://www.dw.com/pt-002/caso-anast\%C3\%A1 cio-matavele-seispol\%C3\%ADcias-condenados-a-pris\%C3\%A3o/a-53863961. 
fatti indussero per l'ennesima volta RENAMO e MDM a fare ricorso. In una drammatica riunione della CNE, il risultato fu approvato con 9 voti a favore e 8 contrari, rischiando, per la prima volta, di rimandare gli elettori alle urne per comprovata frode elettorale ${ }^{57}$. Joseph Hanlon dichiarò alla stampa che quelle del 2019 dovevano essere considerate come le peggiori elezioni della storia del paese, sia per le frodi massicce che per il fatto che più di 3000 osservatori non vennero accreditati, rendendo impossibile il lavoro di conteggio parallelo dei voti che solitamente veniva svolto ${ }^{58}$. Questa volta anche la delegazione in Mozambico dell'Unione Europea individuò numerosi casi di irregolarità sia prima che durante e dopo il processo elettorale, fra cui l'inserimento nelle urne di voti prestampati, il voto multiplo da parte di diversi elettori, la mancata convalida intenzionale di pacchetti di voti a favore delle opposizioni e l'alterazione dei risultati in varie urne, con l'aggiunta di voti ulteriori59. La stessa opinione fu espressa da altri osservatori, che considerarono il ritorno a un'accentuata polarizzazione come dannoso per la democrazia mozambicana ${ }^{60}$.

La gestione post-elettorale - ricorso di rito al tribunale a parte non fu difficile: in primo luogo, il maggior partito di opposizione, la RENAMO, era diviso e il suo leader aveva deciso di intraprendere la strada della pacificazione con il governo, anche per limitare gli effetti potenzialmente devastanti della scissione operata da Nhongo; in secondo luogo, il paese, o almeno gran parte delle sue classi dirigenti, aveva compreso che il percorso di transizione autoritaria era ormai compiuto e doveva essere accettato, con l'integrazione della "Makon-

${ }^{57}$ LUSA, Moçambique: Filipe Nyusi reeleito Presidente com $73 \%$ dos votos, oposição alega fraude, in "Dw", 27/10/2019, https://www.dw.com/pt-002/mo\%C3\%A7ambique-filipe-nyusi-reeleito-presidente-com-73-dos-votos-oposi $\% \mathrm{C} 3 \% \mathrm{~A} 7 \% \mathrm{C} 3 \% \mathrm{~A} 3 \mathrm{o}$-alegafraude/a-51008391.

${ }^{58}$ N. Issufo, M. Leonel, R. DA SiLVA, "Foram as piores eleições de sempre de Moçambique", diz investigador britânico, in "Dw", 17/10/2019, https://www.dw.com/pt002/foram-as-piores-elei $\% \mathrm{C} 3 \% \mathrm{~A} 7 \% \mathrm{C} 3 \% \mathrm{~B} 5$ es-de-sempre-de-mo\%C3\%A7ambique-diz-i nvestigador-brit $\% \mathrm{C} 3 \% \mathrm{~A} 2$ nico/a-50876399.

${ }^{59}$ LUSA, Missão da UE deteta "irregularidades e más práticas" nas eleições moçambicanas, in "Dw", 08/11/2019, https://www.dw.com/pt-002/miss\%C3\%A3o-da-ue-detetairregularidades-e-m $\% \mathrm{C} 3 \% \mathrm{~A} 1 \mathrm{~s}-\mathrm{pr} \% \mathrm{C} 3 \% \mathrm{~A} 1$ ticas-nas-elei $\% \mathrm{C} 3 \% \mathrm{~A} 7 \% \mathrm{C} 3 \% \mathrm{~B} 5 \mathrm{es}-\mathrm{mo} \% \mathrm{C} 3 \%$ A7ambicanas/a-51170551.

${ }^{60}$ M. A. Pitcher, Mozambique Elections 2019: Pernicious Polarization, Democratic Decline, and Rising Authoritarianism, in "African Affairs", 119/476, 2020, pp. 468-486. 
dizzazione" dello Stato; infine, quella "normalizzazione" delle voci dissidenti, soprattutto di quelle degli avversari politici, che era iniziata con Guebuza e aveva assunto sfumature in parte diverse con i Makonde al governo, doveva guardarsi da un fenomeno del tutto imprevisto e che sta rischiando a tutt'oggi di far deflagrare il Mozambico come mai era successo prima: gli attacchi terroristici a Cabo Delgado, contro lo Stato e contro i Makonde (o forse sarebbe meglio dire contro lo Stato dei Makonde), da parte delle maggioranze etniche Kimwani e Amakhuwa che vivono da decenni in quella provincia da diseredati ed emarginati, e che hanno trovato in una ideologia islamica estremista il loro riferimento e il loro nuovo motivo di identità collettiva ${ }^{61}$.

\section{Conclusioni}

Il lungo percorso di transizione all' "inverso", come qui è stata chiamata, da un iniziale modello democratico a uno autoritario, si è sostanzialmente compiuto in Mozambico con le elezioni del 2019. Esse hanno rappresentato la maggiore frode elettorale organizzata nella storia di questo paese, senza peraltro tentare di celare i brogli, le irregolarità e le esclusioni di osservatori indipendenti, sia nazionali che internazionali, arrivando a ucciderne barbaramente anche uno. Le opposizioni, ormai indebolite, hanno stentato a reagire, e i soggetti che, oggi, maggiormente reclamano, diversamente da quanto succedeva fino alle elezioni del 2014 , sono osservatori e partner internazionali, entrambi delusi dal "tradimento" del Mozambico. Se il processo di affermazione dell'autoritarismo (per il momento in salsa Makonde, ma probabilmente, dal 2024, con una diversa configurazione) ha avuto il suo culmine elettorale nel 2019, ciò non significa che esso si sia fermato o stabilizzato. Trattandosi di un processo ai suoi inizi, il consolidamento è in itinere: il Covid-19, lo scandalo del debito pubblico e soprattutto il conflitto a Cabo Delgado hanno contribuito ulteriormente a restringere gli spazi disponibili del dibattito nel paese. Quanto più vi è da nascondere, da parte della classe diri-

${ }^{61}$ Si veda, fra gli altri, S. HabIBE, S. Forquilha, J. PereIRA, Radicalização islâmica no Norte de Moçambique, Maputo, IESE, 2019, in https://www.iese.ac.mz/wp-content/uploads/2019/09/cadernos_17.pdf. 
gente, tanto maggiori sono le tendenze ad accentuare gli elementi di intolleranza verso le voci critiche. E il governo mozambicano ha molto da celare, sia rispetto alle diffuse pratiche di corruzione che verso le gravissime violazioni dei diritti umani sul fronte di Cabo Delgado, con possibili complicità da parte dei politici e militari Makonde rispetto a questa nuova guerra.

Passi in avanti sulla strada della svolta autoritaria sono stati fatti anche di recente: una nuova legge sulla libertà di stampa e di espressione sta per essere approvata dal Parlamento e questo limiterà al massimo la possibilità di avere corrispondenti in territorio mozambicano da parte della stampa internazionale, che in questo momento è quella più attiva su quanto sta accadendo nel nord del paese. Tom Bawker, corrispondente del sito inglese Zitamar, che dava informazioni aggiornate proprio sul fronte della guerra contro i terroristi islamici, è stato espulso dal paese a causa di un permesso di lavoro che non avrebbe mai richiesto al governo mozambicano. Infine, la repressione a volte assai dura contro coloro che contravvengono al coprifuoco obbligatorio decretato a più riprese dal presidente della repubblica per limitare i contagi da COVID-19 (repressione ammessa dallo stesso comandante generale della polizia) costituisce un'altra faccia della costruzione dell'autoritarismo mozambicano in cerca di una nuova ideologia, oltre che di specifiche misure politiche. Una ideologia che sembra definirsi secondo criteri di sovranità nazionale, limitazione delle interferenze esterne e delle libertà fondamentali di espressione da parte dei cittadini mozambicani, militarizzazione dello Stato, specialmente nelle aree urbane, di più facile controllo, e processi elettorali anch'essi largamente "amministrati" per garantire la vittoria al FRELIMO e al suo candidato presidenziale. 


\begin{abstract}
Relying upon the concept of "negative peace" proposed by Galtung, this article aims at showing the deterioration of the Mozambican political framework in the last years, from the first mandate of the President Guebuza (which began in 2009) until the two mandates of Nyusi (elected in 2014 and in 2019). The central thesis is that this situation has its roots in the previous conditions of this country. Among them, the General Peace Agreement signed by Mozambican Government guided by FRELIMO and the main opposition party, RENAMO, played a significant role. In particular, it has not been able to overcome the structural violence which has always connoted the economic, political and ethnic relations in Mozambique since its independence, in 1975. When Mozambique adopted a democratic Constitution in 1990, free and transpar-
\end{abstract}

ent elections had to be used to channel the popular discontent towards an acceptable form of dissent against the dominant party, FRELIMO. However, the lack of transparency in all Mozambican elections reduced this important tool to a mere formal instrument that FRELIMO used to confirm its power. This research focuses on the analysis of the elections held in 2009, 2014 and 2019, on their respective preparation and subsequent management. It shows how a restricted FRELIMO élite, at the beginning headed by Guebuza, then by Nyusi, in representation of Makonde's privileged élite, could provoke an inversion in the process of Mozambique's democratization, which contributed to nourish the old conflict with RENAMO, and to generate a new conflict, in the North of the country, carried out by supposed Islamic terroristic groups. 